\title{
Investigation of the effect of turbulence intensity and nozzle exit boundary layer thickness on stability pattern of subsonic jet
}

\author{
Siavash Gohardehi*, Saeed Arablu, Hossein Afshin, and Bijan Farhanieh \\ Center of Excellence in Energy Conversion, School of Mechanical Engineering, Sharif University of Technology, \\ P.O. Box: 11155-9567, Iran
}

Received: 14 December 2017 / Accepted: 2 September 2018

\begin{abstract}
In this study, factors affecting the noise generation by instability waves in a subsonic jet with acoustic Mach number of 0.5 are investigated using linear stability analysis. The base flow required for instability analysis is obtained by modeling the jet stream based on the $k-\varepsilon$ turbulence model and using the empirical coefficients suggested by Thies and Tam [1]. The resulting base flow profiles are used to solve the linear instability equation, which governs the pressure perturbation for obtaining the eigenvalues and eigenfunctions. The results of linear instability analysis for phase and amplitude of pressure fluctuations are compared against the existing experimental data, which demonstrated the validity of the conducted instability analysis. The effects of turbulence intensity and thickness of the boundary layer at the jet nozzle exit on the results of the linear instability analysis are investigated. The results show that as the turbulence intensity at nozzle exit increases, the frequency range for which the spatial growth rates are positive grows smaller, and except for very low frequencies, this leads to decreased growth rates in both axisymmetric and first azimuthal modes. Also, in both of these modes, an increase in the thickness of the boundary layer at nozzle exit leads to a decrease in perturbation's growth rates in the surveyed frequency ranges.
\end{abstract}

Keywords: Turbulence jet noise / linear stability / turbulence intensity / boundary layer thickness

\section{Introduction}

With the increasing utilization of jet propulsion technology in the recent years, acoustic noise generated by these engines has become a matter of concern. Despite many attempts to reduce the jet noise, for example, using Chevron nozzles [2], steady fluidic injections [3], and plasma discharge actuator [4], the absence of a conceptual framework for expressing the mechanism of noise generation in turbulent jet streams has limited the success of above-mentioned and similar methods. It is therefore evident that further development of the jet noise reduction solutions requires better understanding of physics of the phenomenon and also improved modeling concepts based on an accurate theory [5].

Among many studies performed on the jet noise, the majority has been based on the Lighthill's Acoustic Analogy [6,7] and its generalized forms. In acoustic analogy, noise sources need to be modeled. As the noise of the jet stream is generated by the turbulence, so modeling this kind of noise is far more challenging.

\footnotetext{
* Corresponding author: e-mail: Gohardehi@mech.sharif.edu
}

Until 1970, the turbulence in free shear flows used to be theorized based primarily on incoherent fine-scale turbulence structures, but the extensive experimental evidence provided by Crow and Champagne [8], Brown and Roshko [9], and Moore [10] led to the detection of coherent largescale structures as the dominant cause of jet noise at low frequencies and low angles relative to the jet axis. Coherent large-scale structures, also called instability waves [11], are created by flow instability [12], and in free shear flows, which have an inflection point in their velocity profiles, are considered as Kelvin-Helmholtz instabilities [13,14]. The fluctuation induced by these structures can be modeled with Linear Stability Theory (LST).

In acoustically excited jets, pressure and velocity fluctuations have been successfully predicted using eigenfunctions obtained from linear stability analysis [15-17]. For natural jets, Suzuki and Colonius [18] performed a set of experiments with microphone arrays in a linear hydrodynamic regime to obtain the amplitude and phase of pressure fluctuations produced by instability waves. They compared the results with the results of local linear stability analysis and found an acceptable level of consistency. Parabolized Stability Equations [19] have been used to investigate the effect of nonparallel base flow and study of jet noise in 
several articles [20]. Gudmundsson and Colonius [21] performed a stability analysis based on the modeled base flow of experimental data. They used PSE and LST to obtain the growth rate of the unstable perturbation and pressure fluctuation in the potential core of the subsonic jet. They compared their results with the experimental results of the microphone array and proper agreement has been reported.

As discussed in the earlier researches, the jet nozzle exit boundary condition has a significant effect on the instability features of the jet flow. Bogey et al. [22] surveyed the effect of the nozzle exit turbulence level on the flow and acoustic field of the subsonic jet. They reported longer potential core in case of an increase in nozzle exit turbulence intensity. Bogey and Bailly [23] investigated the effect of the nozzle exit condition on the flow and pressure fluctuation of the jet using large eddy simulation. They found the jet flow field to be highly sensitive to the upstream condition by different simulation on each flow configuration. The main aim of this research is to investigate the effect of the nozzle exit conditions on the flow characteristics by using reduced order models that are governed by linear stability theory.

In the remainder of this article, Section 2 describes the conditions and parameter of simulated jet flow. In Section 3, after introducing the governing equations, the resulting linear stability equations and boundary conditions are provided. To solve the stability equation, base flow needs to be determined, thus the modeling approach and the method of obtaining the base flow profiles are explained, and finally, the numerical method that is used for solving the instability equation is briefly described. In Section 4 , first the numerically obtained base flow results are validated against the existing experimental data; then the results of linear stability analysis for amplitude and phase of pressure fluctuation are compared with the experimental results. Finally, results obtained with different values of turbulence intensity and boundary layer thickness at the nozzle exit are presented and discussed. In Section 5 , the results of the study are summarized.

\section{Flow conditions and parameters}

In this study, flow conditions at the nozzle outlet are based on experimental research of Bridge and Wernet [24] at NASA's Glenn Research Center, and the set point 3 defined by Tanna [25]. Details of this experimental study can be found in reference [24]. These conditions are expressed as follows:

- Acoustic Mach number is 0.5. This number is the ratio of jet speed at nozzle exit to sound speed at far-field region (ambient sound speed),

- Temperature ratio of the jet is 0.95 . This number is the ratio of jet temperature at nozzle exit to air temperature at far-field region (ambient air temperature),

- Nozzle diameter is 2 in.,

- According to the provided data, turbulence intensity at nozzle exit is about $2 \%$,

- Reynolds number is $7.5 \times 10^{5}$,

- Ambient temperature is $288 \mathrm{~K}$,

- Ambient pressure is $1 \mathrm{Atm}$.
Table 1. Different investigated scenarios for turbulence intensity and boundary layer thickness at jet nozzle exit.

\begin{tabular}{ll}
\hline $\begin{array}{l}\text { Turbulence intensity } \\
\text { at nozzle exit }(T i)\end{array}$ & $\begin{array}{l}\text { Boundary layer thickness } \\
\text { at nozzle exit }(\delta)\end{array}$ \\
\hline 2 & 0.085 \\
3.5 & 0.085 \\
5 & 0.085 \\
8 & 0.085 \\
10 & 0.085 \\
2 & 0.225 \\
2 & 0.17 \\
2 & 0.0425 \\
2 & 0.0283 \\
\hline
\end{tabular}

Thickness of the boundary layer at the nozzle exit has not been mentioned in reference [24]. So, to determine this parameter, the experimental results provided in reference [20] are used. One of the experiment cases of this research has the same Mach number, jet temperature, and nozzle diameter as those are assumed in the present study. Hence, the boundary layer thickness reported in that article is used, which is $\delta=0.085$, and has been normalized by the nozzle diameter.

For a fixed Mach number and jet temperature ratio, the parameters of turbulence intensity and boundary layer thickness at the nozzle exit could be changed and adjusted. Therefore, to evaluate the effect of these parameters, the different indicated scenarios in Table 1 are investigated.

\section{Method of solution}

In this research, turbulent compressible circular jet flow is investigated in the cylindrical coordinate system. For simulating the flow, a cylindrical domain with the length of 30 and the radius of 10 (normalized based on nozzle diameter) have been selected. The studied fluid is air, which is assumed to be a Newtonian fluid and satisfy the ideal gas equation of state.

\subsection{Governing equations}

Parameters $x, r$, and $t$, which represent the streamwise and radial direction and time, respectively, are normalized as follows:

$$
x=\frac{x^{*}}{D} ; r=\frac{r^{*}}{D} ; t=\frac{t^{*} a_{\infty}}{D},
$$

where stars symbolize dimensional variables, $D$ represents the nozzle diameter, and $a_{\infty}$ denotes the ambient speed of sound. Flow parameters are normalized as follows:

$$
\rho=\frac{\rho^{*}}{\rho_{\infty}} ; u=\frac{u^{*}}{a_{\infty}} ; T=\frac{T^{*} c_{p}}{a_{\infty}^{2}} ; p=\frac{p^{*}}{\rho_{\infty} a_{\infty}^{2}} .
$$


In equation (2), $\rho, T$, and $p$ denote the density, temperature, and pressure, respectively, and $u$ is the velocity with axial, radial, and azimuthal components denoted by $u_{x}, u_{r}$, and $u_{\theta}$. Also, $\rho_{\infty}$ and $c_{p}$ represent the ambient fluid density and the specific heat at constant pressure, respectively. The compressible flow's governing equations are in the form of equations (3)-(8).

$$
\begin{gathered}
\frac{D \rho}{D t}+\rho \nabla \cdot u=0, \\
\rho \frac{D u_{x}}{D t}=-\frac{\partial p}{\partial x}+\frac{1}{R e} V_{x}, \\
\rho\left(\frac{D u_{r}}{D t}-\frac{u_{\theta}^{2}}{r}\right)=-\frac{\partial p}{\partial r}+\frac{1}{R e} V_{r}, \\
\rho\left(\frac{D u_{\theta}}{D t}+\frac{u_{r} u_{\theta}}{r}\right)=-\frac{\partial p}{\partial \theta}+\frac{1}{R e} V_{\theta}, \\
\rho\left(\frac{D T}{D t}+(\gamma-1) T \nabla \cdot u\right)=\frac{\gamma}{R e} \Phi+\frac{\gamma}{R e P r} \nabla \cdot Q, \\
p=\frac{\gamma-1}{\gamma} \rho T .
\end{gathered}
$$

The above equations represent the continuity (conservation of mass), axial momentum, radial momentum, azimuthal momentum, conservation of energy, and the ideal gas equation of state, respectively. Here, $\gamma$ denotes the ratio of specific heats for air and is assumed to be 1.4. In the above equations, $\frac{D}{D t}, \nabla$, and $\nabla$. denote the material derivative, gradient, and divergence, respectively. $V, \Phi$, and $Q$ denote the viscous terms, viscous dissipation, and heat flux, respectively. Reynolds and Prandtl numbers are defined as follows:

$$
R e=\frac{U_{j} D}{v_{j}} ; \operatorname{Pr}=\frac{\mu_{\infty} c_{p}}{k_{\infty}} .
$$

In the above equations, $U_{j}$ and $v_{j}$ are the jet velocity and the kinematic viscosity of the fluid at the nozzle exit, while $\mu_{\infty}$ and $k_{\infty}$ are the dynamic viscosity and the thermal conductivity of the fluid at the far-field region. Temperature dependence of viscosity is neglected as the experimental jets in our study are near-isothermal [21]. The kinematic viscosity, dynamic viscosity, and thermal conductivity of the fluid are assumed to be constant.

Acoustic Mach number (denoted above by $M_{\infty}$ ) is expressed as follows:

$$
M_{\infty}=\frac{U_{j}}{a_{\infty}} .
$$

\subsection{Linear instability analysis}

In the stability analysis, flow variables are assumed as follows:

$$
q(X, t)=\bar{q}(X)+q^{\prime}(X, t),
$$

where $\bar{q}(X)$ denotes the base flow and is assumed to be time-independent, and $q^{\prime}(X, t)$ denotes the perturbation terms. Substituting the above terms into equations (3)-(8) and ignoring the second-order perturbation terms yields the linear perturbation equations. The next step is using local stability theory, which has been developed for parallel flows but can also be used to approximate nearly parallel flows. Based on the local stability theory, flow parameters are assumed as follows:

$$
q(x, r, \theta, t)=\bar{q}(r)+\hat{q}(r) e^{i(\alpha x+m \theta-\omega t)} .
$$

For further simplification and as we are studying inviscid perturbations, diffusion terms of the above equations is ignored. The result of such simplification is known as Rayleigh stability theory.

The resulting Rayleigh system of equations can be solved for pressure term to obtain the following pressure perturbation equation:

$$
\begin{array}{r}
\frac{1}{r} \frac{d}{d r}\left(\frac{r d \hat{p}}{d r}\right)-\left(\frac{2 \alpha}{\alpha \bar{u}_{x}}-\omega \frac{\partial \bar{u}_{x}}{\partial r}+\frac{\partial \bar{\rho}}{\bar{\rho}}\right) \frac{d \hat{p}}{d r} \\
-\left(\frac{m^{2}}{r^{2}}+\alpha^{2}-\bar{\rho}\left(\alpha \bar{u}_{x}-\omega\right)^{2}\right) \hat{p}=0 .
\end{array}
$$

As the mean flow velocity and density gradient vanishes as $r \rightarrow 0$ and $r \rightarrow \infty$, the boundary conditions of the above equation at two bounds of the solution domain are expressed as follows:

$$
\begin{array}{r}
r \rightarrow 0: \hat{p}(r) \rightarrow J_{m}(\beta r) \text { and } \\
\beta=\sqrt{\bar{\rho}(0) *\left(\alpha \bar{u}_{x}(0)-\omega\right)^{2}-\alpha^{2},} \\
r \rightarrow \infty: \hat{p}(r) \rightarrow H_{m}^{1}(\gamma r) \text { and } \gamma=\sqrt{\omega^{2}-\alpha^{2}} .
\end{array}
$$

In the above equations, $J_{m}$ represents the Bessel functions of the first kind and $m$ th order, and $H_{m}^{1}$ denotes the Henkel functions of the first kind and $m$ th order.

In this study, instabilities of the investigated jet are of the convective type, so flow perturbations grow downstream and instability waves are better to be modeled with spatial stability theory [26]. In this method, $\omega$ is a real number representing the angular frequency of perturbations and is given as an input, and $\alpha$ is the eigenvalue that must be calculated. The latter parameter is a complex number, in which the real part is the perturbations wavenumber and the imaginary part is the spatial growth or decay rate of perturbations. Also, $m$ denotes the azimuthal mode number. 


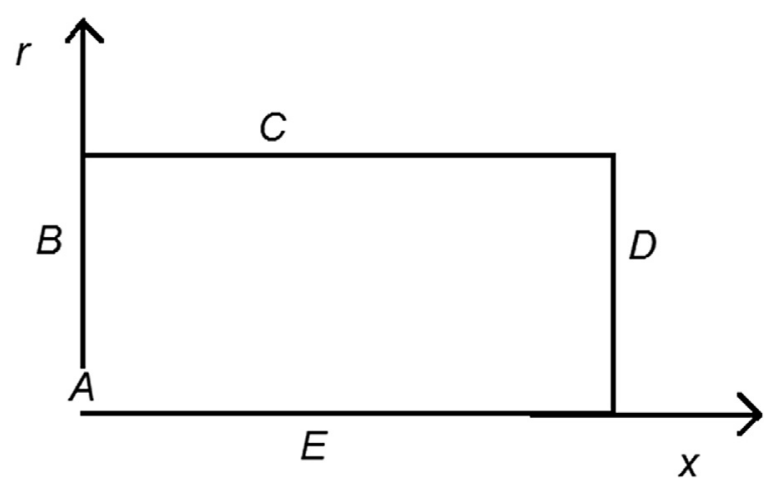

Fig. 1. Schematic of the numerical domain boundary surfaces of the simulation.

\subsection{The base flow model}

The base flow used in stability analysis could be laminar or turbulent. In the jet noise analyses, because of the high speed of jet flow at the nozzle exit, Reynolds number is very high. Based on the nozzle diameter, this number will be of the order of $10^{5}$ or higher [5], and with these Reynolds numbers, the jet flow is turbulent. For turbulent flows, the base flow parameters required for instability analysis are the mean flow parameters that appear in the Reynolds decomposition [21].

For calculating the base flow, a cylindrical domain with the length of 30 and the radius of 10 is selected whose nozzle exit is located on the left lower side and the jet center line is aligned on the cylinder axis. As the surveyed jet is axisymmetric, the two-dimensional rectangle is meshed with structured and orthogonal grids. The radial grids are selected to be finer than axial grids, and as the gradients at nozzle exit region are higher than the domain outlet, this area is constructed with finer mesh compared to the rest of the domain. The schematic of the numerical domain is shown in Figure 1.

Velocity inlet boundary condition is considered for the nozzle exit, which is represented by surface $\mathrm{A}$, temperature condition set to be same as experimental conditions [24]. For other surfaces, i.e., B, C, and D, the boundary conditions are considered as standard pressure and temperature.

Line $\mathrm{E}$ is considered as a jet centerline and defined in the simulating software as an axis boundary condition in the axisymmetric simulation mode.

The mean flow is simulated by ANSYS Fluent software using the $k-\varepsilon$ turbulence model based on experimental coefficients proposed by Thies and Tam [1]. In this simulation, the profile of velocity at the nozzle exit is assumed as the inlet velocity boundary condition of the problem. Following reference [27], the velocity profile is obtained from equation (16), and coefficients of this equation are obtained from equation (17) in terms of acoustic Mach number and boundary layer thickness.

$$
\begin{aligned}
& u_{x}(r)=\operatorname{atanh}(b(1-2 r)) \\
& a=M_{\infty} ; b=\frac{\tanh ^{-1}(0.99)}{2 \delta}
\end{aligned}
$$

Here and in the remainder of this article, all $u$ variables are average values. Turbulence intensity at nozzle exit is also incorporated into the boundary conditions as input. To solve the stability equation, the analytical profiles of axial velocity and density over the entire domain needs to be determined. The axial velocity profile is obtained from equation (18):

$u_{x}(r, x)= \begin{cases}M_{\infty}, & \text { if } r<R(x) \\ u_{c}(x) \exp \left(-(r-R(x))^{N(x)} / b^{N(x)}(x)\right), & \text { otherwise. }\end{cases}$

In equation (18), the values of $u_{c}(x), R(x), N(x)$, and $b(x)$ for each $x$ must be obtained by implementing curve fitting to the results of axial velocity at the point $x$. This equation is similar to the one used by Rodriguez et al. [28], except that in their equation, $N(x)=2$ and is constant, while in this study, to attain a better curve fitting, this parameter is assumed to be variable.

The density profile is obtained using the equation of state and assuming temperature profile as a result of Crocco-Busemann equation as indicated in equation (19).

$$
T=-\frac{u_{x}^{2}}{2}+\left(\frac{1}{\gamma-1}\left(\frac{T_{\text {jet }}}{T_{\infty}}-1\right) \frac{u_{x}}{M_{\infty}}+\frac{M_{\infty}^{2}}{2}\right)+\frac{1}{\gamma-1}
$$

In the above equation, $T_{\text {jet }}$ denotes the flow temperature at nozzle exit.

\subsection{Solving the eigenvalue problem}

The equation of pressure perturbation, equation (13), is a nonlinear eigenvalue problem, which should be solved using the shooting method. To solve this problem, the secondorder equation is converted into two first-order equations. Then, values of $m, \omega$, and profiles $\bar{u}_{x}$ and $\bar{\rho}$ (for each fixed $x$ ) are used as inputs to determine the $\alpha$ value and also satisfying boundary conditions (14) and (15). This $\alpha$ is the eigenvalue, and its corresponding $\hat{p}(r)$ is the eigenfunction. The shooting algorithm first approximates the eigenvalue, and then evolves over radial direction using the fourth-order Runge-Kutta method to calculate the values of pressure functions and their derivatives at the outlet boundary condition. It then uses the values of boundary conditions at these points to update the approximated eigenvalue using the Secant formula. This algorithm repeats the above process until eigenvalue converges. Special care needs to be taken in a singular point of equation (13) where $\alpha \bar{u}_{x}-\omega=0$, and the path of integration must be deformed to avoid the singularity.

\section{Results and discussion}

\subsection{Validation of the base flow model}

To validate the results of the base flow model, in Figures 2 and 3 , the graphs of axial velocity and kinetic energy of the turbulence in the jet centerline are compared with the experimental results of reference [24] (note that modeling 


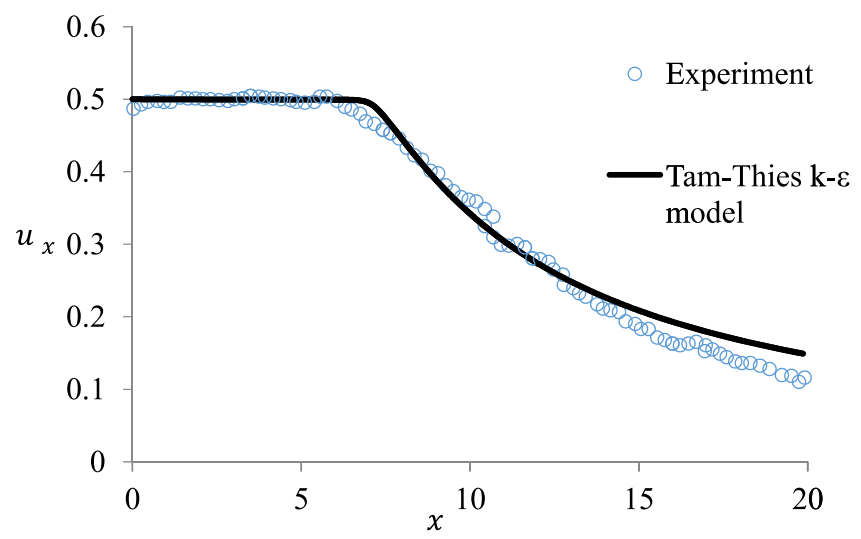

Fig. 2. Comparison of axial velocity of the model jet at centerline with experiment results [24].

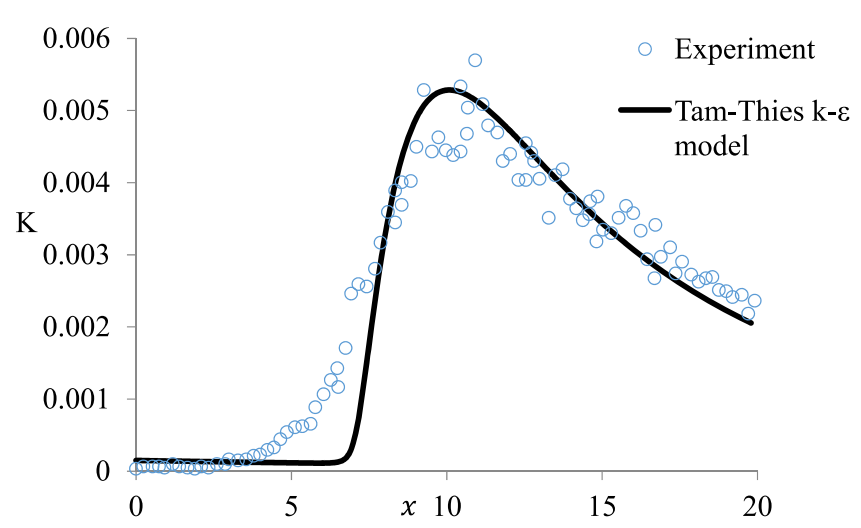

Fig. 3. Comparison of turbulence kinetic energy at jet centerline with experiment results [24].

results belong to $T_{\mathrm{i}}=2 \%$ ). As illustrated in these figures, the modeling results have a good agreement with the experiment. It should be mentioned that the model slightly overestimates the potential core length, where this issue has been thoroughly discussed in [29].

Figure 4 shows the graph of axial velocity in the jet centerline for different turbulence intensities at the nozzle exit. Figures 4 and 5 indicate that as the turbulence intensity at nozzle exit increases, the length of the potential core decreases. Meanwhile, the length of the potential core is defined with the point where the shear layer reaches the jet axis. It can be explained that any increase in the turbulence intensity at the nozzle exit of jet leads to escalations of mixing and interaction of the jet with its environment, which leads to increased momentum transfer from the jet to its surroundings and also causes faster decline of the jet centerline velocity and consequently lower length of the potential core.

A change in the jet centerline velocity and the potential core length means a change in the velocity profile at different cross-sections, which alters the results of the instability analysis. The profile of axial velocity along the radial direction at the cross-section $x=3$ is shown in Figure 5. According to this figure, as turbulence intensity at the nozzle exit increases, so does thickness of the shear layer, and this leads to a reduction of the

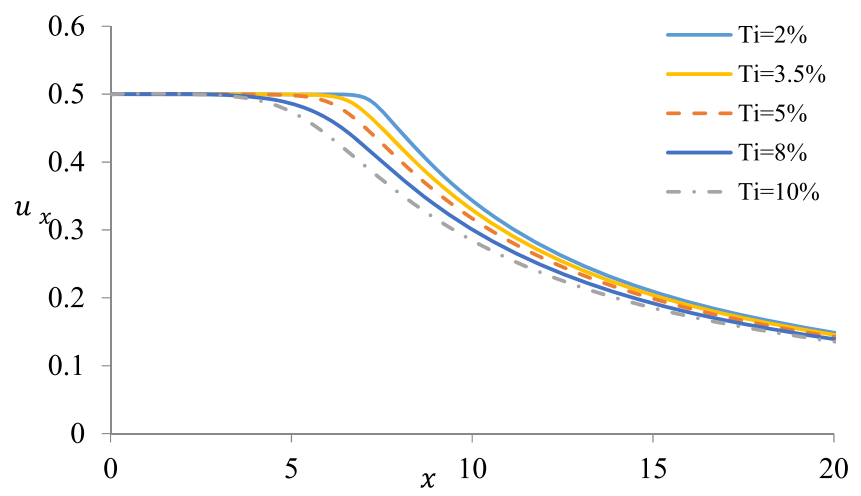

Fig. 4. Axial velocity at jet centerline for different turbulence intensities.

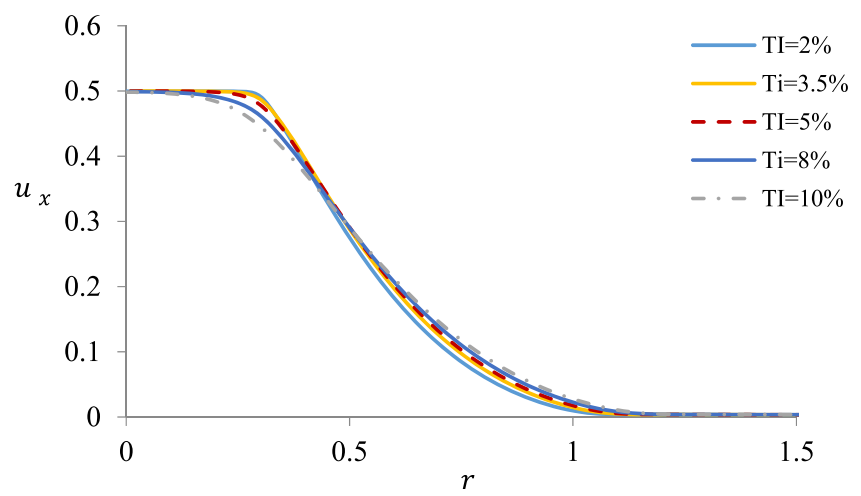

Fig. 5. Axial velocity profile at $x=3$ for different turbulence intensities.

average radial gradient of axial velocity, which has a significant effect on the results of stability analysis. This effect is investigated at the end of this section. Figure 6 shows the shear layer developing in streamwise direction inside the potential core. The thickness of the shear layer has been defined based on the radial distance between two velocity profiles, i.e., $u_{x} / U j=0.01$ and $u_{x} / U j=0.99$. It is clear that as the flow develops in streamwise direction, the thickness of shear layer increases and the higher turbulence intensity leads to the thicker shear layer with a slower growth rate. This slower growth rate for higher turbulence intensity is in consistence with the findings in reference [22].

Figure 7 illustrates the effect of the thickness of the boundary layer at nozzle exit on the base flow profiles. According to this figure, an increase in the thickness of the boundary layer at the nozzle exit leads to a decrease in the potential core length. It could be described that the thickness of the boundary layer at nozzle exit is equal to the thickness of shear layer at the source point of the jet. As this thickness increases, the larger initial thickness of the shear layer allows it to reach the centerline at shorter distance. Therefore, the increase in boundary layer thickness and the consequent increase in initial thickness of the shear layer lead to a decrease in the length of the potential core. 


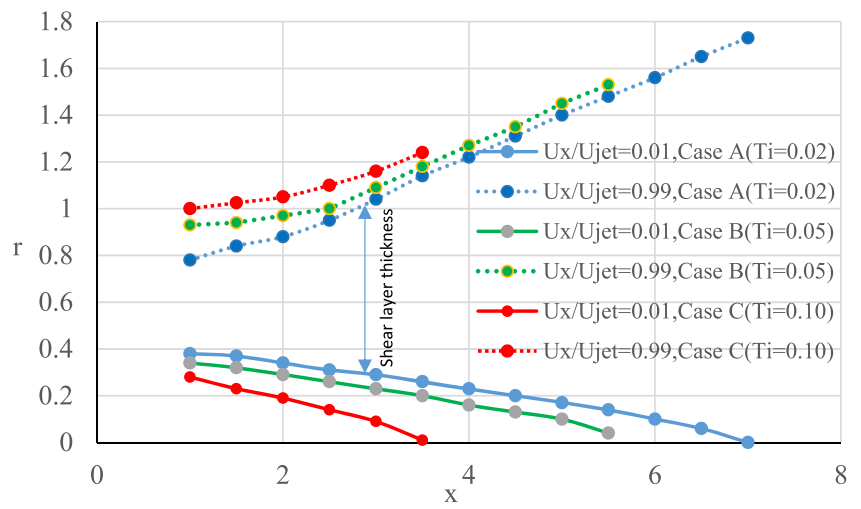

Fig. 6. Shear layer growth in the streamwise direction for different turbulence intensities.

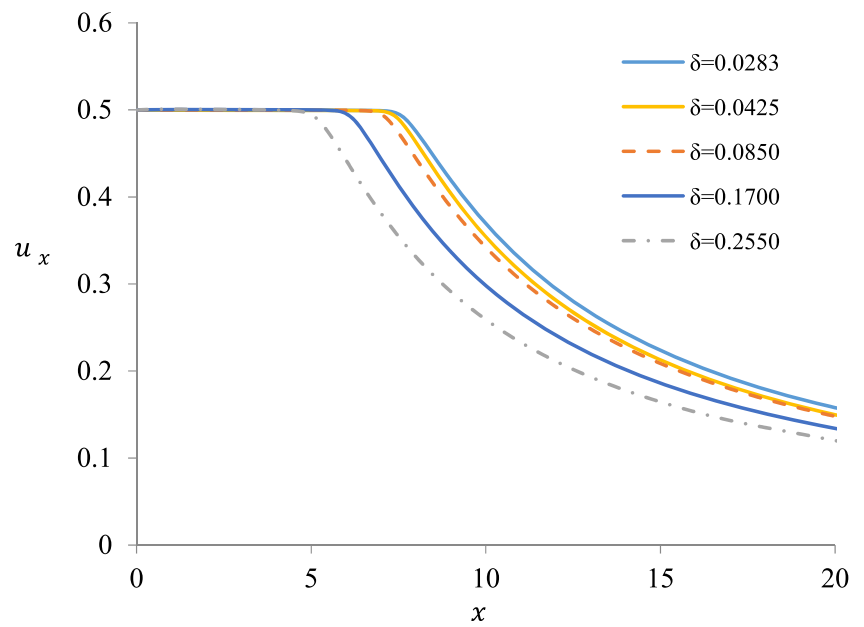

Fig. 7. Axial velocity at jet centerline for different boundary layer thicknesses.
As already mentioned, any change in the jet centerline velocity and the potential core length changes the velocity profile at different cross-sections. Another example of this effect is shown in Figure 8, which illustrates the profile of axial velocity for the cross-section $x=3$. Figure 8 shows that, for the reason recounted earlier, the thickness of the shear layer increases with the increase in the thickness of the boundary layer.

\subsection{Validation of the linear stability analysis}

To validate the results of the linear stability analysis, pressure perturbations obtained with this method are compared with the experimental results of Suzuki and Colonius [18]. They conducted a series of experiments with microphone arrays that were located in the linear hydrodynamic region and provided the pressure fluctuations at the locations of microphones in terms of Fourier and azimuthal modes, which are represented in the form of equation (20) (for more detail, see Ref. [18]).

$$
p^{\prime}(x, r, \theta, t)=\sum_{m} \int \tilde{p}_{m \omega}(x, r) e^{i(m \theta-\omega t)} d \omega
$$

Suzuki and Colonius reported the amplitude and phase of $\tilde{p}_{m \omega}(x, r)$ at the location of microphones, which precisely provides a global expression of modes.

To compare the results of the local linear stability analysis with the globally expressed experimental results, local modes should be converted to global modes. For this purpose, first, eigenfunctions obtained for different crosssections $(x)$ must be normalized. In this regard, eigenfunctions obtained for all modes and all cross-sections $(x)$ are assumed to have a maximum amplitude of unity and phase of zero at $r=0.5$. Pressure fluctuations in the global modes are expressed with equation (20), and the global

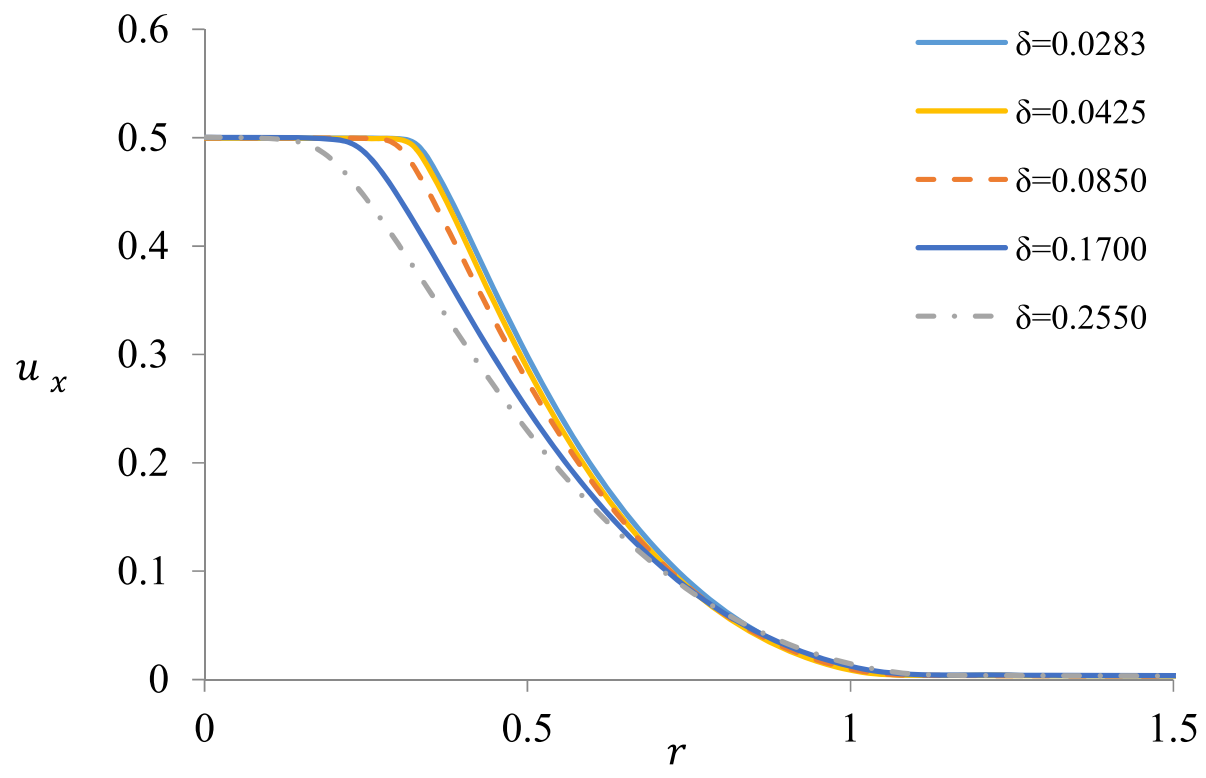

Fig. 8. Axial velocity at $x=3$ for different boundary layer thicknesses. 
eigenfunctions $\tilde{p}_{m \omega}(x, r)$ are obtained based on the local eigenfunctions by using equation (21).

$$
\tilde{p}_{m \omega}(x, r)=\hat{p}_{m \omega}(r, x) e^{i \int_{x_{0}}^{x} \alpha_{m \omega}(\eta) d \eta}
$$

In equation $(21), \hat{p}_{m \omega}(r, x)$ represents the normalized version of the eigenfunctions obtained for each mode using the local stability analysis, and $\alpha_{m \omega}$ denotes the eigenvalues obtained for each mode using the local stability analysis. $x_{0}$ is an arbitrary value, which we assume to be zero. In this equation, $\hat{p}_{m \omega}(r, x)$ is highly dependent on radial component $r$ and less so on $x$. In fact, dependence of $\tilde{p}_{m \omega}(x, r)$ on $r$ is based on the function $\hat{p}_{m \omega}(r, x)$, while its dependence on $x$ is based on the function $e^{i \int_{x_{0}}^{x} \alpha_{m \omega}(\eta) d \eta}[18]$.

When using linear stability analysis to obtain the pressure perturbations, it should be evoked that equation (13) is homogeneous, so any combination of the solution is itself a solution. This means that perturbations obtained with linear stability analysis are not exact solution and for each particular mode, they can be multiplied by an arbitrary number. Similarly, the phases obtained for each mode can be summed with a constant. So, to compare our results against the experimental results, pressure perturbation function is assumed to be in the form of equation (22).

$$
p^{\prime}(x, r, \theta, t)=\sum_{m} \int A_{m \omega} \hat{p}_{m \omega}(r, x) e^{i \int_{x_{0}}^{x} \alpha_{m \omega}(\eta) d \eta} e^{i(m \theta-\omega t)} d \omega
$$

In equation (22), the amplitude and phase of the complex coefficient $A_{m \omega}$ are assumed such that the results of stability analysis show a good agreement with the microphone data of reference [18].

In Figures 9-12, the amplitude and phase obtained for each mode from the linear stability analysis (for $T_{\mathrm{i}}=2 \%$ ) are compared with the experimental data. The illustrated stability analysis results are in fact the amplitude and phase $i \int^{x} \alpha_{m \omega}(\eta) d \eta$

of $A_{m \omega} \hat{p}_{m \omega}(r, x) e^{x_{0}}$

$$
\int^{x} \alpha_{m \omega}(\eta) d \eta
$$

the microphones are laid [18].

As is shown in Figures 9-12, the results of the linear stability analysis have a good agreement with the experimental results. Also, Strouhal number is defined as $S t=\omega / 2 \pi M_{\infty}$.

\subsection{The effect of turbulence intensity at the nozzle exit on the results of linear stability analysis}

The growth rates, $-\alpha_{i}$, in terms of frequency for the axisymmetric mode and the first azimuthal mode for different turbulence intensity levels are shown, respectively, in Figures 13 and 14. According to these figures, as turbulence intensity at the nozzle exit increases, the

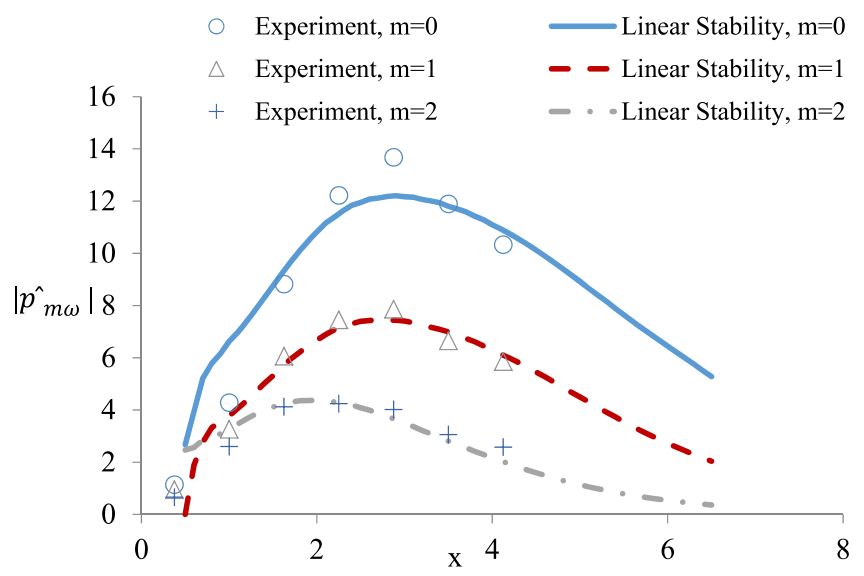

Fig. 9. Validation of amplitude of pressure fluctuation at St $=0.35$ for different azimuthal modes $(m=0,1$, and 2$)$ with results of reference [18].

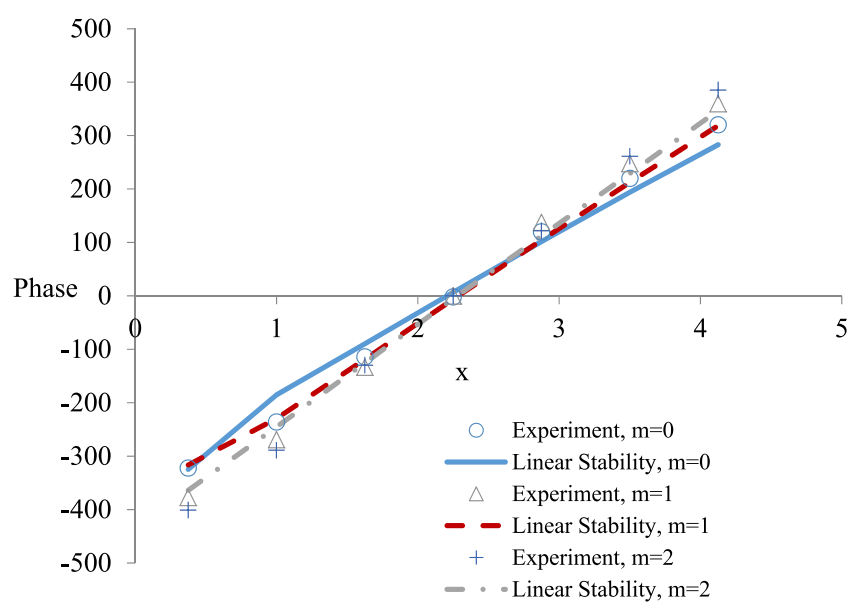

Fig. 10. Validation of phase of pressure fluctuation at $\mathrm{St}=0.35$ for different azimuthal modes with results of reference [18].

frequency range for which the growth rate is positive grows smaller, and except for very low frequencies, the increased turbulence intensity leads to a decreased growth rate for both mentioned modes. The reasons for this reduction is that improved turbulence intensity means faster momentum transfer from jet to its environment, which leads to an increased growth rate of the shear layer and its increasing thickness in all cross-sections. The increased thickness of the shear layer reduces the radial gradient of the axial velocity, which results in the reduced growth rate. Comparing axisymmetric modes and first azimuthal modes reveals that the maximum growth rate in first azimuthal modes occurs at the perturbations with lower frequency. The higher frequency axisymmetric and first azimuthal modes become more stable by increasing turbulence intensity. It is presented in the mentioned figures that for the frequency range higher than $\mathrm{St}=0.8$, increasing turbulence intensity leads to damping the unstable perturbations. 


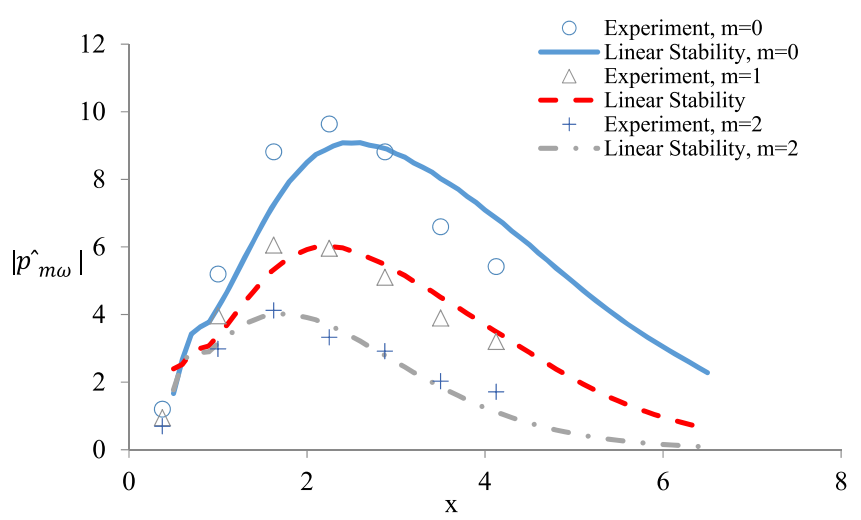

Fig. 11. Validation of amplitude of pressure fluctuation at $\mathrm{St}=0.5$ for different azimuthal modes with results of reference [18].

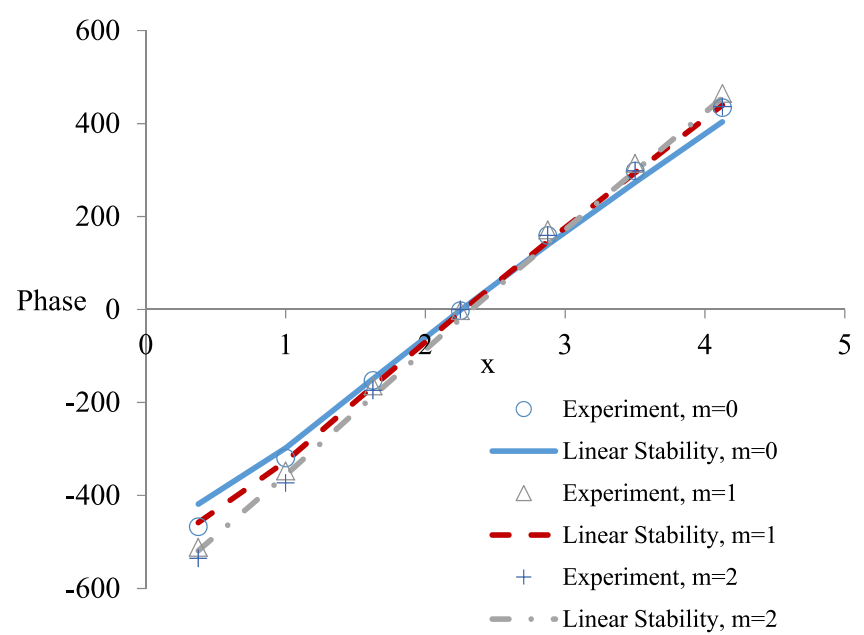

Fig. 12. Validation of phase of pressure fluctuation at $\mathrm{St}=0.5$ for different azimuthal modes with results of reference [18].

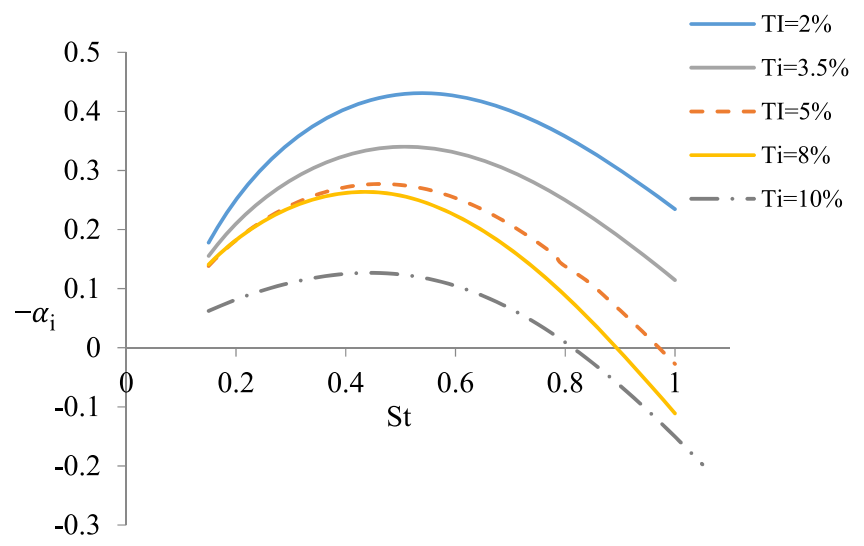

Fig. 13. Growth rate of axisymmetric mode $(m=0)$ for different turbulence intensity at $x=3$.

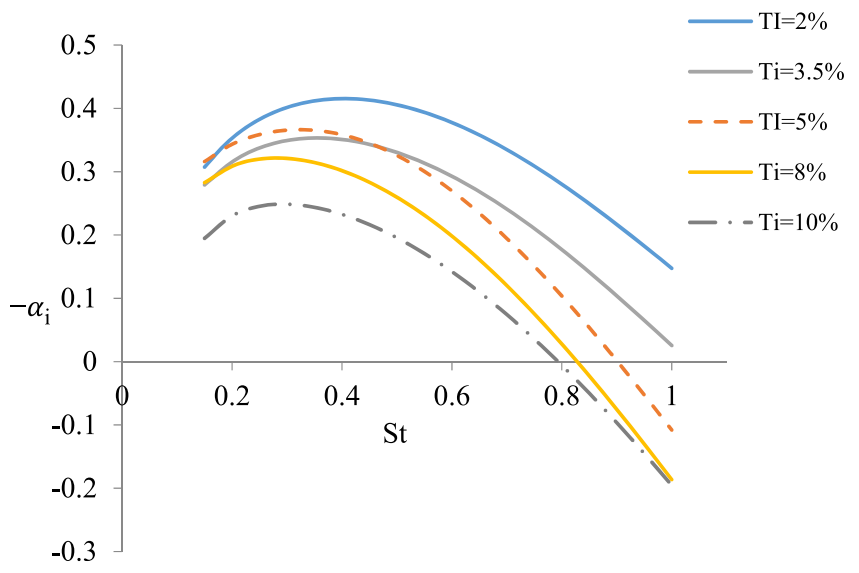

Fig. 14. Growth rate of first azimuthal mode $(m=1)$ for different turbulence intensity at $x=3$.

In Figure 15, growth rate and wave number variation in streamwise direction are shown for axisymmetric modes for different Strouhal number. It is seen that maximum wave number increases as the turbulence intensity increases. For higher Strouhal numbers, maximum wave number happens in the shorter axial position. Maximum growth rates are reported at the vicinity of the nozzle and by marching in streamwise direction, the growth rate reduces rapidly. Increasing turbulence intensity has a reducing effect on the growth rate in the streamwise locations with $x>3$. Furthermore, by increasing turbulence intensity, the wave number is increased despite the reduction in growth rate. It is worth to note that for $T_{i}=10 \%$, as the potential core has been found shorter, the growth rate for axisymmetric mode tends to zero after $x=4$, which is termination point of the potential core.

Figure 16 presents the growth rate and wave number variation in streamwise direction for first azimuthal modes for different Strouhal number. It is seen that for lower Strouhal numbers, the wave number increases in streamwise direction. In the higher frequency perturbations, the wave number has a peak value in the axial location with $x<2$ and the maximum wave number increases as the turbulence intensity increases. As in Figure 15, the maximum growth rates are reported in the vicinity of the nozzle and by marching in streamwise direction, the growth rate reduces rapidly. Increasing turbulence intensity has a reducing effect on the growth rate in the axial locations with $x>2$. It is worth to note that for $T_{i}=10 \%$, as discussed earlier and despite the stable axisymmetric modes, there are spatially growing helical modes in the locations outside the potential core.

\subsection{The effect of boundary layer thickness at the nozzle exit on the results of linear stability analysis}

The spatial growth rates of perturbations in terms of frequency for the modes $m=0$ and $m=1$ and different boundary layer thicknesses (at nozzle exit) are shown in 

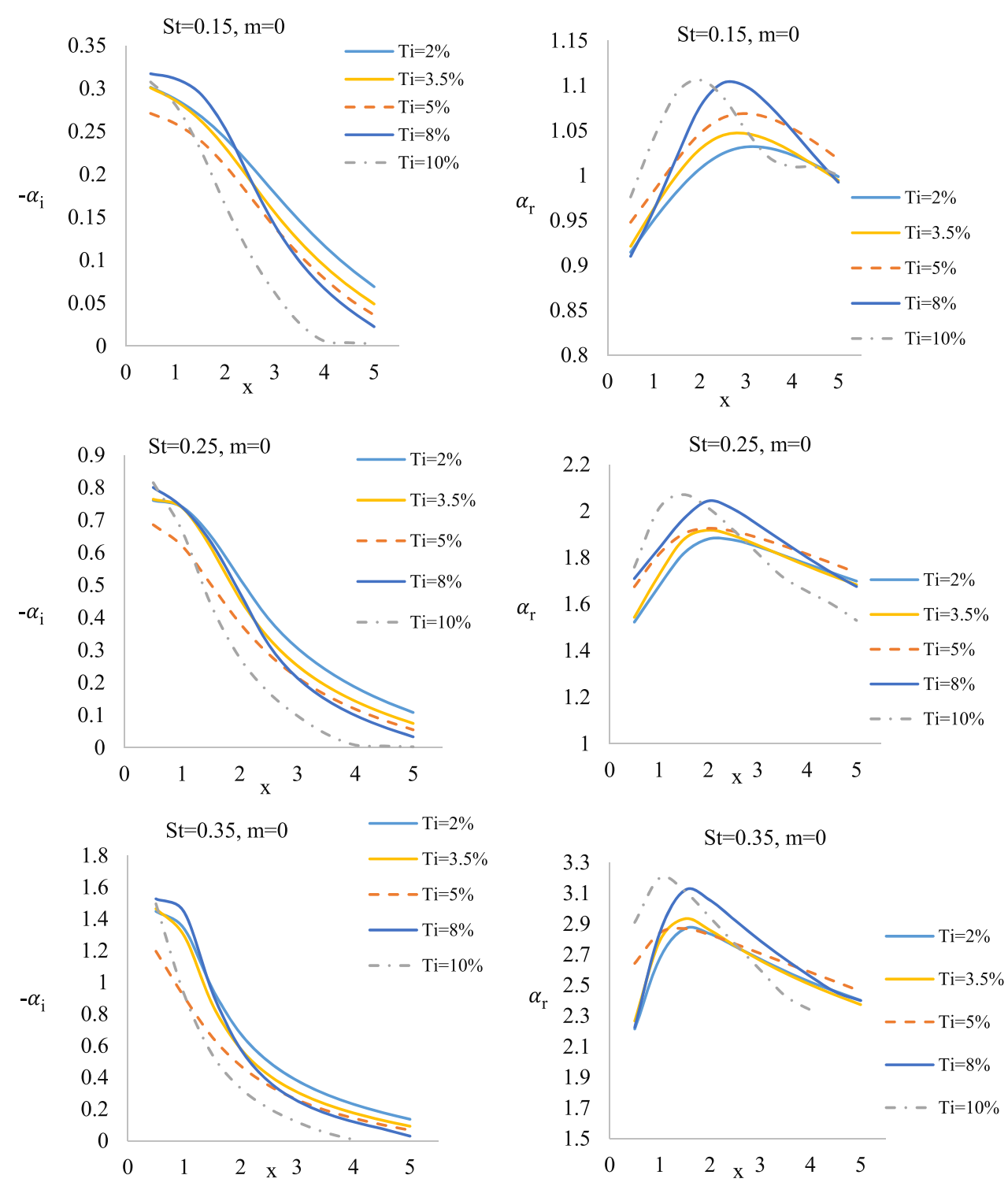

Fig. 15. Growth rate $\left(-\alpha_{i}\right)$ and wave number $\left(\alpha_{r}\right)$ of the axisymmetric modes $(m=0)$ for different turbulence intensity at St $=0.15$, 0.25 , and 0.35 .

Figures 17 and 18. According to these figures, for both axisymmetric and first azimuthal mode, as the boundary layer thickness at the nozzle exit rises, perturbation growth rate decreases. The reason is explained based on the increase in jet shear layer thickness and consequently decrease in the radial gradient of axial velocity, which leads to lower growth rates.

It is seen that the maximum growth rate for all cases occurs at $\mathrm{St}=0.55$ for axisymmetric modes and at $\mathrm{St}=0.45$ for fist azimuthal modes. Also higher boundary layer thickness causes perturbations to become stable at the lower frequencies.

In Figure 19, the imaginary and real part of the axisymmetric modes is illustrated for different Strouhal numbers in streamwise direction. It is found that as the boundary layer thickness increases, the growth rate decreases and the wave number increases. By thickening the boundary layer, the maximum wave number shifts to the locations near the nozzle exit. It is worth to note that in streamwise locations with $x<1$, the boundary layer thickness variation has not much effect on the growth rate and this effect intensifies in the locations far downstream of the nozzle. In the lower Strouhal number, the maximum wave number for thinner boundary layer occur at the location far downstream of the nozzle and by increasing the Strouhal number, the maximum wave number moves to the lower axial location.

Figure 20 presents the growth rate and the wave number for first azimuthal modes in streamwise direction. For these modes, the effect of the boundary layer thickening on the reducing growth rate is smaller than the axisymmetric modes and this affect is damped 

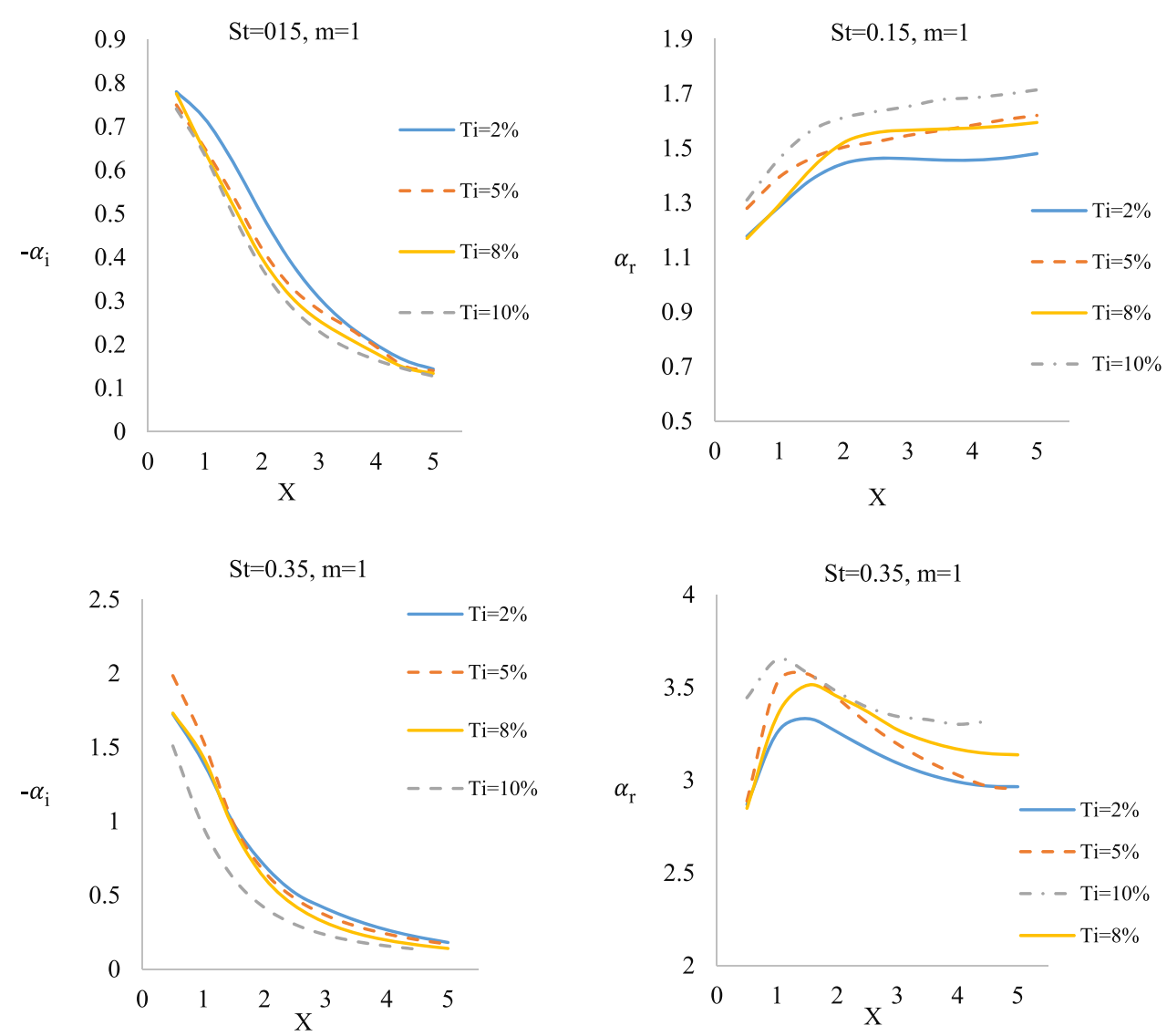

Fig. 16. Growth rate $\left(-\alpha_{i}\right)$ and wave number $\left(\alpha_{r}\right)$ of the first azimuthal modes $(m=1)$ for different turbulence intensity at St $=0.15$ and $\mathrm{St}=0.35$.

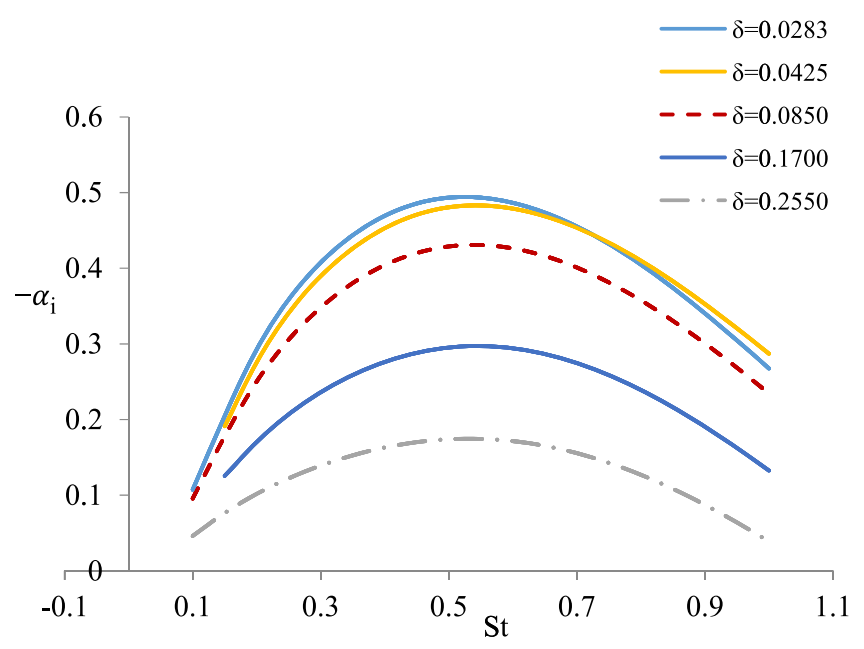

Fig. 17. Growth rate of axisymmetric mode $(m=0)$ for different jet exit boundary layer thickness at $x=3$.

for the locations with $x>5$. The thicker boundary layer leads to strengthen decrease in the growth rate in the streamwise direction. Also, for higher frequency modes, the wave number variation is limited for $x>2$. In the axial positions smaller than $x=2$, as the boundary

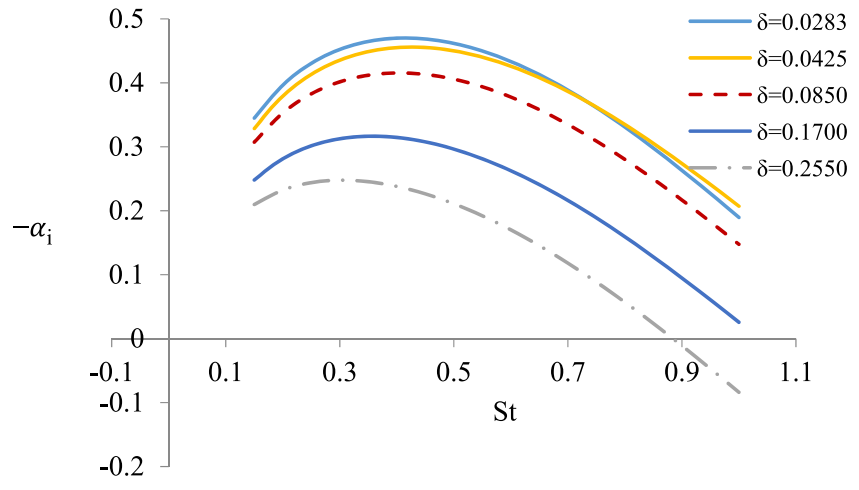

Fig. 18. Growth rate of first azimuthal mode $(m=1)$ for different jet exit boundary layer thickness at $x=3$.

layer thickness increases, the wave number increases and its maximum value location moves closer to the nozzle exit. It is interesting that for lower Strouhal number, the wave number increases in the streamwise direction. 

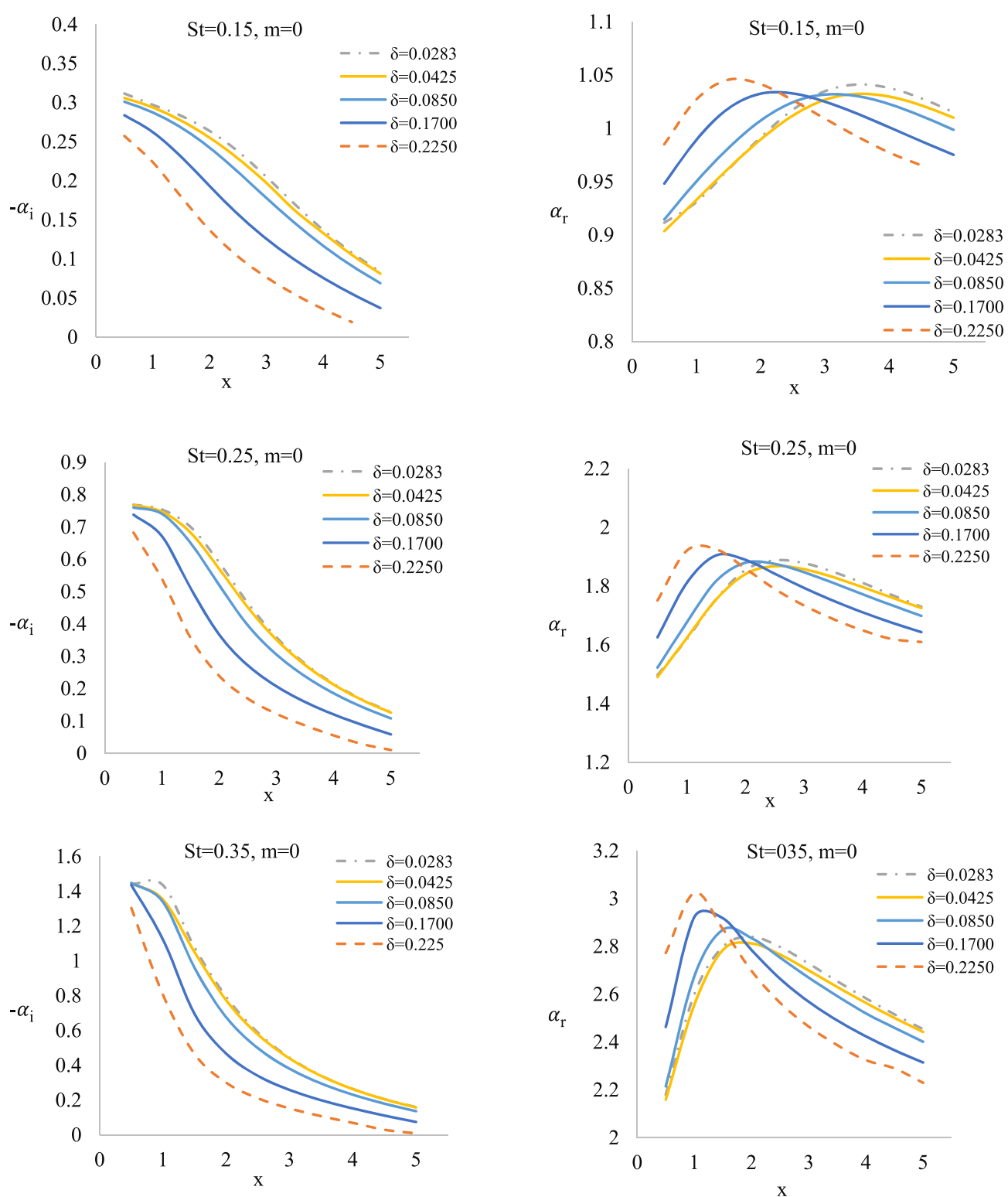

Fig. 19. Growth rate $\left(-\alpha_{i}\right)$ and wave number $\left(\alpha_{r}\right)$ of the axisymmetric $(m=0)$ for different boundary layer thicknesses at St $=0.15$, 0.25 , and 0.35 .

\section{Conclusion}

In this study, the pressure fluctuation generated by instability waves of a subsonic jet with acoustic Mach number of 0.5 is investigated by the linear stability analysis. Comparison of the amplitude and phase obtained with this method against the experimental data demonstrated the validity of the results. Next, the effects of two parameters, turbulence intensity and thickness of the boundary layer at the nozzle exit, on the results of linear stability analysis are surveyed.

The results show that as turbulence intensity at the nozzle exit rises, the frequency range for which the spatial growth rate is positive (unstable perturbations) shrinks, and except for very low frequencies, the increased turbulence intensity leads to the decreased growth rate for both axisymmetric and first azimuthal modes. The reason behind this reduction is that the increased turbulence intensity leads to faster momentum transfer from jet to its environment, which itself leads to a growth of the shear layer and its growing thickness in all cross-sections. The increased thickness of the shear layer consequently decreases the radial gradient of the axial velocity, which results in lower growth rate. The wave number increases by intensifying the turbulence intensity at the nozzle exit and for the higher Strouhal numbers, the wave number's peak values move closer to the nozzle exit.

According to the results, as the boundary layer thickness at the nozzle exit increases, in both axisymmetric and first azimuthal modes, the perturbation growth rate in the surveyed frequency range decreases. The wave number becomes higher by thickening the boundary layer. It is 

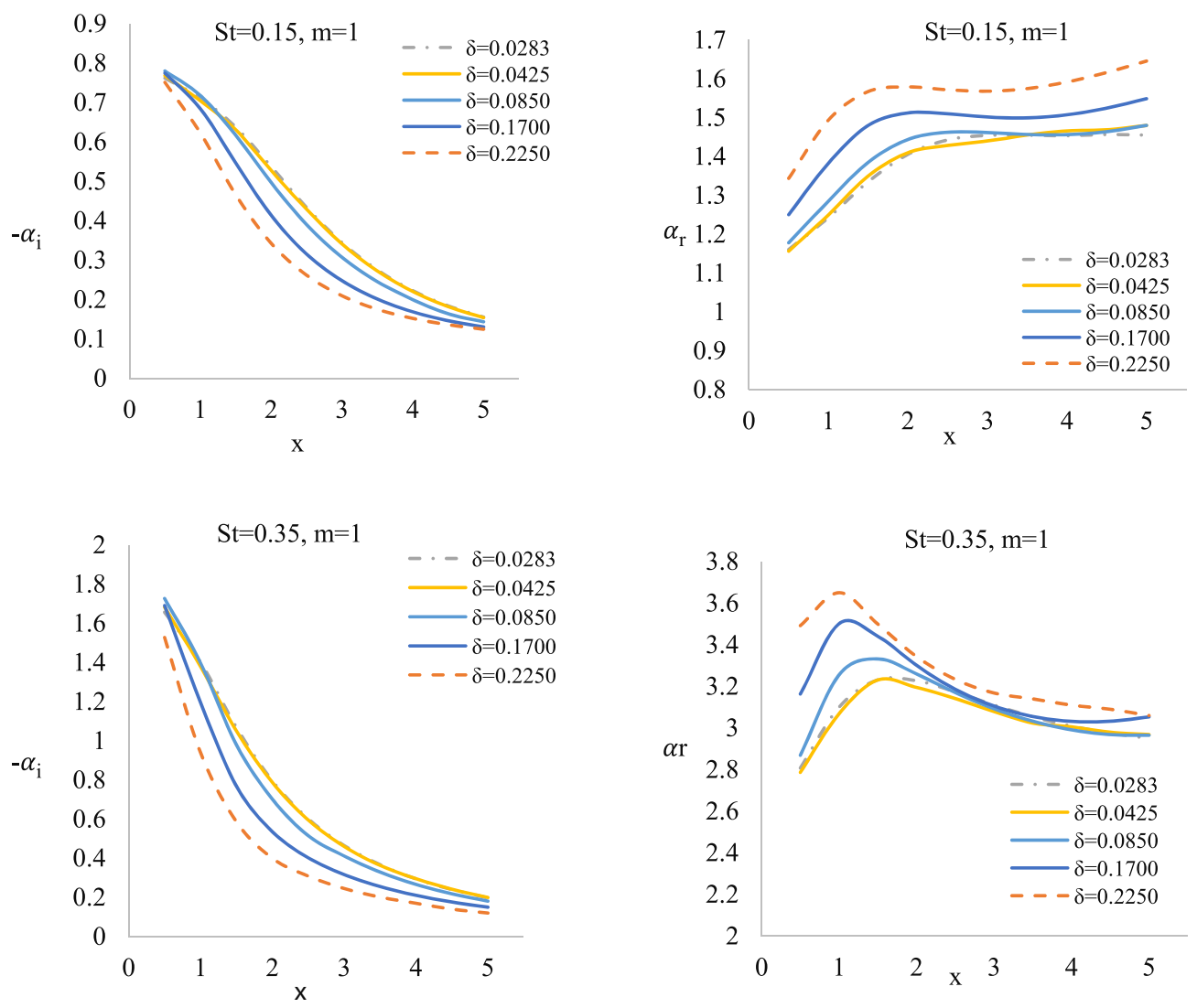

Fig. 20. Growth rate $\left(-\alpha_{i}\right)$ and wave number $\left(\alpha_{r}\right)$ of the first azimuthal modes $(m=1)$ for different boundary layer thicknesses at $\mathrm{St}=0.15$ and $\mathrm{St}=0.35$.

explained that the thickness of the shear layer at the source point of the jet equals to the thickness of the boundary layer at nozzle exit. Therefore, growing of the thickness of the boundary layer leads to further thickening of the shear layer with the jet development. The increase in thickness of the shear layer reduces the radial gradient of the axial velocity, which leads to a reduced perturbation growth rate.

\section{References}

[1] A.T. Thies, C.K. Tam, Computation of turbulent axisymmetric and nonaxisymmetric jet flows using the k-epsilon model, AIAA J. 34 (1996) 309-316.

[2] K. Zaman, J. Bridges, D. Huff, Evolution from 'tabs' to 'chevron technology'- a review, Int. J. Aeroacoustics 10 (2011) 685-709.

[3] B. Henderson, Fifty years of fluidic injection for jet noise reduction, Int. J. Aeroacoustics 9 (2010) 91-122.

[4] M. Samimy et al., Active control of a Mach 0.9 jet for noise mitigation using plasma actuators, AIAA J. 45 (2007) 890901.

[5] P. Jordan, T. Colonius, Wave packets and turbulent jet noise, Ann. Rev. Fluid Mech. 45 (2013) 173-195.

[6] M.J. Lighthill, On sound generated aerodynamically. I. General theory, Proc. R. Soc. London Ser. A Math. Phys. Sci. 211 (1952) 564-587.
[7] M.J. Lighthill, On sound generated aerodynamically. II. Turbulence as a source of sound, Proc. R. Soc. London A: Math. Phys. Eng. Sci. (1954). The Royal Society.

[8] S.C. Crow, F. Champagne, Orderly structure in jet turbulence, J. Fluid Mech. 48 (1971) 547-591.

[9] G.L. Brown, A. Roshko, On density effects and large structure in turbulent mixing layers, J. Fluid Mech. 64 (1974) $775-816$.

[10] C. Moore, The role of shear-layer instability waves in jet exhaust noise, J. Fluid Mech. 80 (1977) 321-367.

[11] C.K. Tam, Jet noise: since1952, Theor. Comput. Fluid Dyn. 10 (1998) 393-405.

[12] A.F. Hussain, Coherent structures - reality and myth, Phys. Fluids (1958-1988) 26 (1983) 2816-2850.

[13] D. Crighton, M. Gaster, Stability of slowly diverging jet flow, J. Fluid Mech. 77 (1976) 397-413.

[14] R. Mankbadi, J. Liu, A study of the interactions between large-scale coherent structures and fine-grained turbulence in a round jet, Philos. Trans. R. Soc. London Ser. A Math. Phys. Sci. 298 (1981) 541-602.

[15] K. Zaman, A. Hussain, Vortex pairing in a circular jet under controlled excitation. Part 1. General jet response, J. Fluid Mech. 101 (1980) 449-491.

[16] C.K. Tam, P.J. Morris, The radiation of sound by the instability waves of a compressible plane turbulent shear layer, J. Fluid Mech. 98 (1980) 349-381.

[17] R. Petersen, M. Samet, On the preferred mode of jet instability, J. Fluid Mech. 194 (1988) 153-173. 
[18] T. Suzuki, T. Colonius, Instability waves in a subsonic round jet detected using a near-field phased microphone array, J. Fluid Mech. 565 (2006) 197-226.

[19] T. Herbert, Parabolized stability equations, Annu. Rev. Fluid Mech. 29 (1997) 245-283.

[20] A.V. Cavalieri et al., Wavepackets in the velocity field of turbulent jets, J. Fluid Mech. 730 (2013) 559-592.

[21] K. Gudmundsson, T. Colonius, Instability wave models for the near-field fluctuations of turbulent jets, J. Fluid Mech. 689 (2011) 97-128.

[22] C. Bogey, O. Marsden, C. Bailly, A computational study of the effects of nozzle-exit turbulence level on the flow and acoustic fields of a subsonic jet, in 17th AIAA/CEAS Aeroacoustics Conf., 2011.

[23] C. Bogey, C. Bailly, Influence of nozzle-exit boundary-layer conditions on the flow and acoustic fields of initially laminar jets, J. Fluid Mech. 663 (2010) 507-538.
[24] J. Bridges, M.P. Wernet, Measurements of the aeroacoustic sound source in hot jets, AIAA paper 3130 (2003) 2003.

[25] H. Tanna, An experimental study of jet noise Part I: Turbulent mixing noise, J. Sound Vib. 50 (1977) 405-428.

[26] P. Huerre, P.A. Monkewitz, Absolute and convective instabilities in free shear layers, J. Fluid Mech. 159 (1985) $151-168$

[27] X. Garnaud et al., The preferred mode of incompressible jets: linear frequency response analysis, J. Fluid Mech. 716 (2013) 189-202.

[28] D. Rodríguez et al., A study of linear wavepacket models for subsonic turbulent jets using local eigenmode decomposition of PIV data, Eur. J. Mech. B Fluids 49 (2015) 308-321.

[29] N.J. Georgiadis, J.R. DeBonis, Navier-Stokes analysis methods for turbulent jet flows with application to aircraft exhaust nozzles, Prog. Aerospace Sci. 42 (2006) 377-418.

Cite this article as: S. Gohardehi, S. Arablu, H. Afshin, B. Farhanieh, Investigation of the effect of turbulence intensity and nozzle exit boundary layer thickness on stability pattern of subsonic jet, Mechanics \& Industry 20, 103 (2019) 